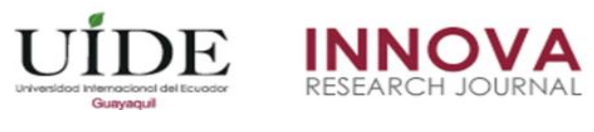

INNOVA Research Journal, ISSN 2477-9024

(Junio, 2017). Vol. 2, No.6 pp. 83-93

DOI: https://doi.org/10.33890/innova.v2.n6.2017.216

URL: http://revistas.uide.edu.ec/index.php/innova/index

Correo: innova@uide.edu.ec

\title{
Impacto del uso de dinero electrónico en estudiantes universitarios
}

\section{Impact of use of electronic money on university students}

\author{
Ernesto Novillo Maldonado \\ Universidad Técnica de Machala, Ecuador \\ Miguel Espinosa Galarza \\ Universidad Técnica de Machala, Ecuador \\ María Palomeque Solano \\ Universidad Técnica de Machala, Ecuador
}

Autor por Correspondencia: enovillo@utmachala.edu.ec, mespinosa@utmachala.edu.ec, maeupaso@gmail.com

Fecha de recepción: 29 de Marzo de 2017 - Fecha de aceptación: 5 de Junio de 2017

Resumen: El presente trabajo se fundamenta en la aplicación de estadística descriptiva para estudiar el impacto que tiene el dinero electrónico en el Ecuador por parte de los estudiantes. El objetivo de la investigación es conocer la percepción de los estudiantes sobre el uso del dinero electrónico como medio de pago. La metodología usada se basó en revisiones bibliográficas para tener las bases necesarias sobre el dinero electrónico, de la misma manera se aplicó un enfoque cuantitativo, a través del cuestionario personal. Posteriormente se llevó a cabo el procesamiento de datos con el software SPSS, para realizar un análisis descriptivo. Dentro de los resultados obtenidos se destaca que el 65,4\% de la muestra considera factible el uso de dinero electrónico, otro de los resultados encontrados es que existe un desconocimiento de las ventajas y desventajas de su uso, pues el 39,8\% de la muestra tenía conocimiento del tema. Finalmente se tiene que los estudiantes universitarios conocen o han escuchado sobre el dinero electrónico. Pero existe cierta falencia debido a que no conocen las ventajas y desventajas del mismo.

Palabras claves: dinero; dinero electrónico; sistema de pago; política monetaria

\begin{abstract}
The present work is based on the application of descriptive statistics to study the impact of electronic money on Ecuador by students. The objective of the research is to know the students' about the perception of the use of electronic money as a means of payment. The methodology used was based on bibliographical revisions to have the necessary bases on the electronic money, in the same way a quantitative approach was applied, through the personal questionnaire. Subsequently the data processing was carried out with the SPSS software, to perform a descriptive analysis. Among the obtained results it is highlighted that $65.4 \%$ of the sample considers the use of electronic money feasible, another of the results found is that there is an ignorance of the advantages and disadvantages of its use, since $39.8 \%$ of The sample had knowledge of the subject. Finally you have that university students know or have heard about electronic money. But there is a certain failure because they do not know the advantages and disadvantages of it.
\end{abstract}

Key words: money; electronic money; payment system; monetary policy 


\section{Introducción}

El mundo en el que vivimos, el dinero se ha convertido en uno de los cimientos de nuestra sociedad, es parte esencial de una economía de intercambio. En épocas pasadas existían grupos que no los necesitaban para realizar sus actividades, por ejemplo los grupos aislados que viven de la recolección y de la caza. Pero para la gran mayoría es esencial para manejarse en el ámbito económico. Siendo considerado como uno de los grandes avances de la humanidad.

En la actualidad mundial el dinero electrónico ha tenido un aumento importante en su uso, en especial el denominado dinero plástico (tarjetas de crédito, débito, transacciones por internet y monederos electrónicos) y esta tendencia dirigida hacia este tipo de dinero sugiere reformas en la politica monetaria a mediano plazo. Existen dos motivos que generan el apogeo del uso de dinero electronico. Unos de los puntos es que los usuarios prefieren este medio de pago por la comodidad y seguridad que le otorga este tipo de dinero. Otro de los motivos son las facilidades que brindan a los organismos de control para ejercer su trabajo con relación a los movimientos mometarios realizados por los ciudadanos (Jeftanovic, 2001).

Existen estudios que revisan la situacion ecuatoriana referente al dinero electrónico. Algunos hacen recopilación del proceso de implementación del dinero electronico en el Ecuador, como medio de pago, como herramienta de inclusión financiera y las implicaciones que este tiene (Cevallos, 2014; Miranda, 2015; Grijalva, 2014). En este caso la aplicación en este país viene dada por intermedio del estado, que es el encargao de coordinar los distintos aspectos relacionado a su aoperatividad, pues en la mayoria de países que cuentan con este medio de pago es regularizado por sistemas privados (Méndez, 2015).

En el mundo, la utilización del dinero electrónico como un medio de pago se ha vuelto tan común como el uso del papel moneda (Eliott, 2014). La implementación y uso del dinero electrónico como una opción para medios de pago en el Ecuador, es un tema que aún está en debate, aunque en varios países del mundo ya se lo utiliza (UDLA, 2016). Además la adopción de nuevas reformas financieras y monetarias adoptadas por países del Sur en respuesta ala inestabilidad económica ha generado nuevas propuestas que buscan una integración regional (Legeard, 2011).

El dinero electrónico es el valor monetario equivalente al valor expresado en la moneda de curso legal del país (dólares de los Estados Unidos de América), que se intercambia principalmente a través de dispositivos electrónicos, móviles (Valencia, 2015). Para tratadistas destacados es realmente es un medio de pago, nada más que eso, solo una forma moderna, rápida, efectiva, barata y segura de hacer pagos (Quiroga, 2016).

Sin embargo a pesar de la publicidad y marketing con que han contado los nuevos medios de pago, es cuanto menos sorprendente que los métodos tradicionales sean todavía los más populares. Las nuevas tecnologías permiten asegurar que el coste de procesar una transacción adicional utilizando uno de estos nuevos instrumentos es menor que el coste marginal de realizar una transacción utilizando uno de los métodos tradicionales. Además, vale la pena recalcar que los sistemas de pago electrónico son potencialmente más eficientes que los métodos de pago tradicionales o que aquellos basados en papel, y en muchos casos, incluso son más seguros (Santomá, 2001). 
Hay que hacer una diferenciación conceptual entre Dinero Electrónico y el Sistema de Dinero Electrónico implementado en el Ecuador que en terminos del propio Banco Central del Ecuador (2014) menciona:

Es el conjunto de: operaciones, mecanismos, procedimientos y normativas que facilitan los flujos, almacenamiento y transferencias en tiempo real, entre los distintos agentes económicos, a través del uso de: dispositivos electrónicos, electromecánicos, móviles, tarjetas inteligentes y otros que se incorporen producto del avance tecnológico.

Los participantes del Sistema de Dinero Electrónico son todos aquellos actores, personas naturales y jurídicas que voluntariamente han decidido interactuar con el administrador, para ofrecer su infraestructura tecnológica y para realizar transacciones monetarias y no monetarias. Todos los participantes organizan su funcionamiento con base en el Reglamento de Participantes y el Manual de Operaciones emitidos por el BCE, así como a los convenios suscritos (BCE, 2014).

1- Emisor y Administrador.

2- Entidades reguladoras.

3- Canales tecnológicos - Operadoras telefónicas fijas y móviles, operadores satelitales, operadores eléctricos, operadores TV, otros.

4- Macroagentes - empresas, organizaciones e instituciones públicas y privadas; instituciones financieras y del sistema popular y solidario; que en su modelo de negocio mantienen una red de establecimientos de atención al cliente y están en la capacidad de adquirir dinero móvil, distribuirlo o convertirlo en especies monetarias conforme los procedimientos que establecidos por el BCE.

5- Centros transaccionales - Todas las oficinas de atención de los Macroagentes, directas o corresponsales.

6- Personas

En este ultimo grupo personas considerados como actores claves dentro del sistema de dinero electronico se encuentran inmiscuidos los Estudiantes Universitarios, elementos principales de nuestro trabajo de investigación, en cuanto al uso y conocimiento que ellos tienen sobre el dinero electronico. Teniendo en consideración que muchos investigadores han tratado sobre el dinero electronico, pero no existen estudios de tipo cuantitativo que ayuden a reforzar hipotesis. En este caso la Hipotesis planteada es que los estudiantes universitarios conocen sobre el dinero electronico, sus ventajas y desventajas.

El objetivo de la presente investigación es conocer la percepción de los estudiantes sobre el uso del dinero electrónico como medio de pago. Para esto usaremos una investigación bibliográfica y la estadística descriptiva para conocer lo que piensan los estudiantes encuestados. Teniendo un estudio de caso que toma a los estudiantes de la Universidad Técnica de Machala.

El conocimiento por parte de la población sobre el dinero electrónico, medio de pago que está considerado en Ecuador, es uno de los problemas que se tiene. Como se manifestó 
previamente existen algunas investigaciones que se hacen con relación al dinero electrónico, pero al no existir la parte cuantitativa se genera la necesidad de conocer la percepción de la población, en este caso a través de los estudiantes universitarios que son parte de la muestra se tendrá una visión que permita tomar acciones de comunicación en caso de ser necesarias.

\section{Metodología}

La presente investigación usó un enfoque cuantitativo, el mismo que tiene como objetivo determinar la apreciación de los estudiantes universitarios con respecto al dinero electrónico; para esto se utilizó el cuestionario como instrumento, debido que este sirve para recolectar la información necesaria, y cuantificar esta data obtenida (Arribas, 2004).

La población objeto de estudio fueron los estudiantes universitarios de carreras administrativas (economía, administración, contabilidad, comercio internacional, marketing y turismo) de la Universidad Técnica de Machala, a la misma que se aplicó a un tamaño de muestra de 246 estudiantes. El tipo de muestreo fue estratificado, debido a que se dividió a la muestra según la proporción de estudiantes por carrera, y a estas muestras se aplicó de manera aleatoria el instrumento (Casal \& Mateu, 2003).

El cuestionario fue elaborado tomando todas las consideraciones de la investigación de mercados concernientes a las preguntas, escalas, y recomendaciones para el éxito del mismo. Se realizó una validación del instrumento a través de un pretest el mismo que fue aplicado con la finalidad de asegurar que las preguntas sean adecuadas, tanto en su forma como en el número. Posteriormente se realizó el trabajo de campo. Para el procesamiento de datos se usó el software estadístico SPSS. Una vez compilada la data necesaria se procedió con el análisis descriptivo de los ítems más relevantes del cuestionario.

\section{Resultados y Discusión}

Lo que se desea saber en esta investigación es si los estudiantes tienen conocimiento sobre el dinero electrónico, para esto se revisan los resultados de las preguntas que ayudan a comprobar la hipótesis planteada. Uno de los primeros resultados se obtuvo referente al conocimiento sobre el dinero electrónico, los resultados se observan en la Tabla 1 y Figura 1.

Tabla 1

¿Conoce sobre el dinero electrónico?

Válido Frecuencia Porcentaje Porcentaje válido Porcentaje acumulado

\begin{tabular}{lllll}
\hline SI & 214 & $87 \%$ & 87,0 & 87,0 \\
NO & 32 & $13 \%$ & 13,0 & 100,0
\end{tabular}




\begin{tabular}{llll}
\hline Total & 246 & $100 \%$ & 100,0
\end{tabular}

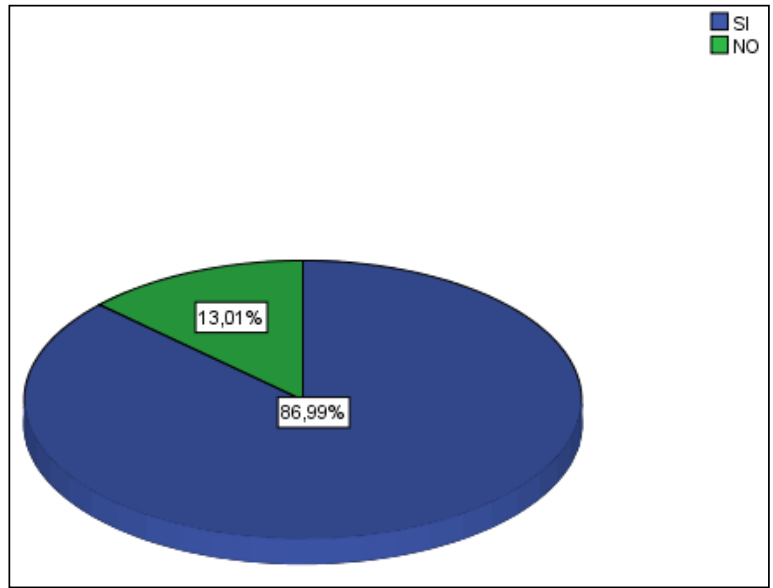

Figura 1. ¿Conoce sobre el dinero electrónico?

De los estudiantes encuestados de las carreras administrativas 214 respondieron que si han escuchado o saben sobre el dinero electrónico, lo cual representa el $87 \%$ del total; lo que indica que la mayor parte de la población estudiantil conoce sobre el dinero electrónico.

Otro de los aspectos importantes a conocer fue lo relacionado a los medios de comunicación que han servido para dar a conocer sobre el dinero electrónico, teniendo los resultados respectivos en la Tabla 2 .

Tabla 2

Medios por los que se ha escuchado o conocido sobre el dinero electrónico

\begin{tabular}{llll}
\hline Medios De Comunicación & \multicolumn{2}{c}{ Respuestas } & Porcentaje de casos \\
\cline { 2 - 3 } Televisión & $\mathrm{N}$ & Porcentaje & \\
Enlace Ciudadano & 155 & $39,10 \%$ & $73,10 \%$ \\
Internet & 65 & $16,40 \%$ & $30,70 \%$ \\
Volantes & 100 & $25,30 \%$ & $47,20 \%$ \\
Prensa Escrita/Revistas & 38 & $9,60 \%$ & $17,90 \%$ \\
Radio & 12 & $3,00 \%$ & $5,70 \%$ \\
Total & 26 & $6,60 \%$ & $12,30 \%$ \\
\hline
\end{tabular}

Para conocer los medios que han servido para divulgar sobre el dinero electrónico, se usó una pregunta de opción múltiple, de la cual se obtuvo que 155 estudiantes contestaron 
seleccionaron televisión, 65 por medio de los enlaces ciudadanos, 100 a través de internet, 12 por medio de volantes, 38 por prensa escrita y revistas y 26 por medio de radio. Esto indicó que el $80,8 \%$ de los medios usados se dio por la televisión con un $39,1 \%$, el internet con el $25,3 \%$ y los enlaces ciudadanos con el 16,4\%.

Otra de las preguntas del cuestionario fue sobre el conocimiento de las ventajas y desventajas del uso del dinero electrónico, de la cual se obtuvieron los resultados de la Tabla 3 y Figura 2.

Tabla 3

\begin{tabular}{lllll}
\multicolumn{4}{c}{ Conocimiento sobre las ventajas y desventajas del uso del dinero electrónico } \\
\cline { 2 - 5 } Válido & Frecuencia & Porcentaje & Porcentaje válido & Porcentaje acumulado \\
\hline SI & 97 & $39,4 \%$ & $39,8 \%$ & $39,8 \%$ \\
NO & 147 & $59,8 \%$ & $60,2 \%$ & $100,0 \%$ \\
Total & 244 & $99,2 \%$ & $100,0 \%$ & \\
Perdidos en el Sistema & 2 & $0,8 \%$ & & \\
Total & 246 & $100,0 \%$ & \\
\hline
\end{tabular}

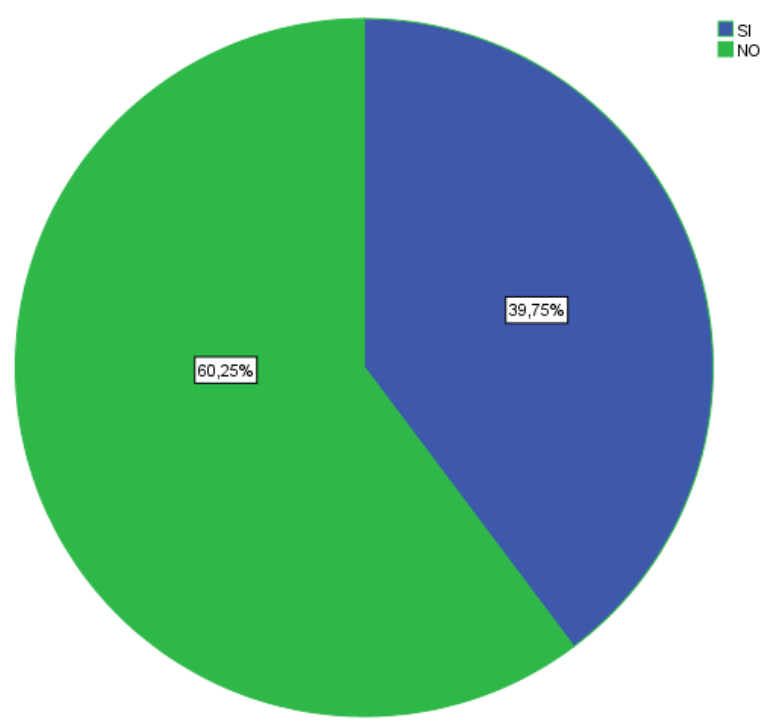

Figura 2. Conocimiento sobre las ventajas y desventajas del uso del dinero electrónico

Como resultado acerca del conocimiento de las ventajas y desventajas del uso del dinero electrónico en los estudiantes universitarios de la Universidad Técnica de Machala se obtuvo que 97 del total de encuestados respondieron que si conocen las ventajas y desventajas, lo cual equivale al 39,4\%; lo que nos indica que se deben plantear estrategias para poder llegar con la información necesaria a todos los ciudadanos que tendrían la posibilidad de usar el dinero electrónico. 
En la Tabla 4 y Figura 3 se observa los resultados concernientes a la factibilidad del uso de dinero electrónico en el Ecuador.

Tabla 4

Factibilidad del uso de dinero electrónico

\begin{tabular}{lcccc}
\hline Válido & Frecuencia & Porcentaje & Porcentaje válido & Porcentaje acumulado \\
& & & & \\
\hline SI & 159 & $64,6 \%$ & $65,4 \%$ & $65,4 \%$ \\
NO & 84 & $34,1 \%$ & $34,6 \%$ & $100,0 \%$ \\
Total & & & & 0,0 \\
& 243 & $98,8 \%$ & $100,0 \%$ & \\
Perdidos en el Sistema & 3 & $1,2 \%$ & & \\
& & & & \\
Total & 246 & $100,0 \%$ & &
\end{tabular}

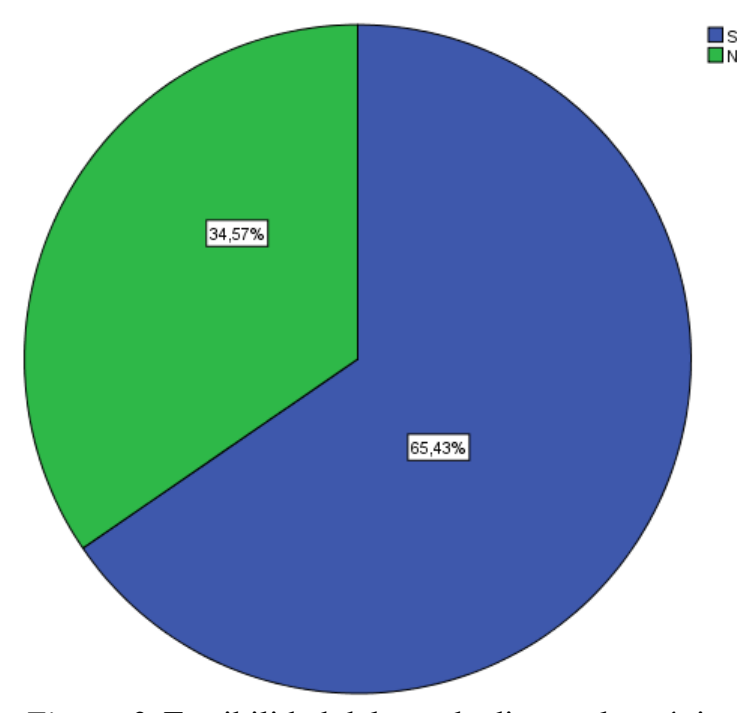

Figura 3. Factibilidad del uso de dinero electrónico

Del total de encuestados se alcanzó que 159 estudiantes, que representan el 64,6\% consideran factible el uso del dinero electrónico en Ecuador, mientras que un $34,1 \%$ piensan todo lo contrario.

Una de las últimas preguntas que se presenta en el cuestionario es para saber si los encuestados creen que el gobierno debería promocionar más y mejor el uso del dinero electrónico. Los resultados se observan en la Tabla 5 y Figura 4. 
Tabla 5

¿Cree usted que el gobierno debería promocionar más y mejor el uso del dinero electrónico?

\begin{tabular}{lllll}
\hline Válido & Frecuencia & Porcentaje & Porcentaje válido & Porcentaje acumulado \\
& & & & \\
\hline SI & 192 & $78,0 \%$ & $78,4 \%$ & $78,4 \%$ \\
NO & 53 & $21,5 \%$ & $21,6 \%$ & $100,0 \%$ \\
Total & 245 & $99,6 \%$ & $100,0 \%$ & \\
Perdidos en el Sistema & 1 & $0,4 \%$ & & \\
Total & 246 & $100,0 \%$ & & \\
& & & & \\
\hline
\end{tabular}

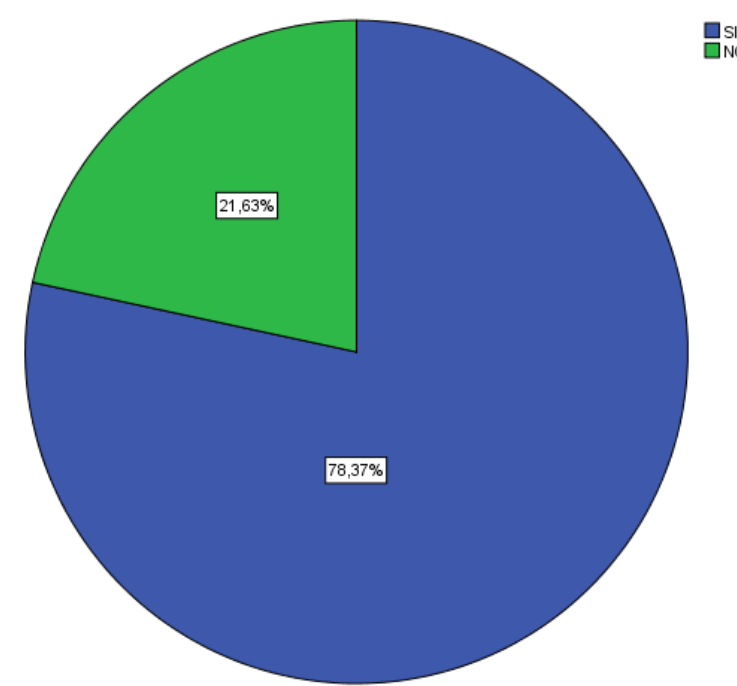

Figura 4. ¿Cree usted que el gobierno debería promocionar más y mejor el uso del dinero electrónico?

Del total de encuestados 192 que es equivalente al 78\% respondieron que "SI", es decir que consideran que debe existir una mayor promoción, y comunicación acerca del dinero electrónico y su uso por parte del gobierno. Lo cual se esperaba como resultado debido a que la pregunta relacionada con el conocimiento sobre las ventajas y desventajas del uso del dinero electrónico de la Tabla 3 nos dio como resultados que la mayor parte de la población no conocía sobre las ventajas y desventajas.

La Tabla 6 nos muestra la tabulación cruzada entre las respuestas sobre el conocimiento de ventajas y desventajas del uso de dinero electrónico y las carreras de los estudiantes.

Tabla 6

Tabulación cruzada entre Ventajas y desventajas del uso del dinero electrónico *Carrera

\begin{tabular}{llll}
\hline Carrera & Conoce Las Ventajas Y Desventajas \\
\cline { 2 - 4 } & SI $\%$ SI & NO & $\%$ NO
\end{tabular}




\begin{tabular}{lccccc}
\hline Economía & 17 & $70,8 \%$ & 7 & $29,2 \%$ & 24 \\
Contabilidad & 39 & $45,9 \%$ & 46 & $54,1 \%$ & 85 \\
Administración & 18 & $29,0 \%$ & 44 & $71,0 \%$ & 62 \\
Hotelería Y Turismo & 4 & $25,0 \%$ & 12 & $75,0 \%$ & 16 \\
Marketing & 1 & $3,7 \%$ & 26 & $96,3 \%$ & 27 \\
Comercio Internacional & 18 & $60,0 \%$ & 12 & $40,0 \%$ & 30 \\
Total & 97 & & 147 & & 244 \\
\hline
\end{tabular}

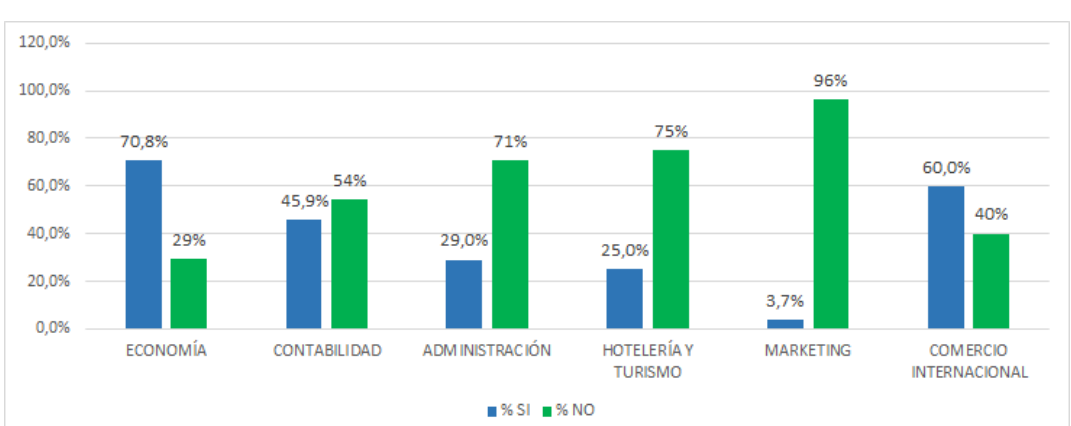

Figura 5. Tabulación cruzada entre Ventajas y desventajas del uso del dinero electrónico*Carrera

En estos últimos resultados se observa que de las carreras seleccionadas en la investigación a nivel general no conocen los estudiantes las ventajas y desventajas del dinero electrónico, pero con los de economía y comercio internacional es la excepción, pues la mayor parte de los estudiantes de estas carreras indicaron que si conocen sobre las ventajas y desventajas del uso de dinero electrónico, en este caso el 70,8\% y el 60\% respectivamente. Por el hecho de que estas carreras revisan estos temas dentro de las distintas asignaturas que les imparten, mientras que en las otras carreras no se enfocan de gran manera en esta temática.

Es importante destacar que como limitante esta investigación fue aplicada a estudiantes de carreras administrativas porque se consideran que son los que más deberían conocer sobre este asunto. Y se aplicó este estudio de caso en la Universidad Técnica de Machala, por tener las facilidades para acceder a esta población. Esta investigación por otro lado es una aplicación de la investigación de mercados, tomando en consideración las consideraciones necesarias para garantizar un instrumento de calidad y a su vez se tomó en consideración los pasos de la investigación de mercados, entre ellos el pretest y la revisión de expertos para garantizar la validez del mismo 


\section{Conclusiones}

Los estudiantes de carreras administrativas de la Universidad Técnica de Machala conocen sobre el dinero electrónico, este conocimiento básico se ha dado a través de televisión, internet y los enlaces ciudadanos. Pero existe cierta falencia debido a que no conocen las ventajas y desventajas del mismo, por consiguiente sería necesario que exista mayor publicidad y se dé a conocer a la ciudadanía más a fondo de este tema. Por lo tanto esto implicaría que se acepta la hipótesis planteada dentro del proyecto. La mayor parte de los estudiantes no poseen el debido discernimiento sobre los uso del dinero electrónico.

De acuerdo a los resultados obtenidos, existe mayor cantidad de estudiantes que considera que es factible el uso del dinero electrónico dentro del país, sin embargo existe una gran cantidad que consideran que el uso de dinero electrónico dentro del país no es factible.

De todas las carreras muestreadas la carrera de economía y comercio internacional son las que tienen mayor conocimiento sobre ventajas y desventajas del dinero electrónico, debido a que estas carreras revisan estos temas dentro de las distintas asignaturas que ven en sus estudios y tienen mayor enfoque de la temática.

\section{Bibliografía}

Arribas, M. (2004). Diseño y validación de cuestionarios. Matronas profesión, 5(17), 23-29.

Banco Central del Ecuador. (Enero de 2014). Banco Central del Ecuador. Recuperado el 2017 de Enero de 27, de Banco Central del Ecuador: www.bce.fin.ec

Casal, J., \& Mateu, E. (2003). Tipos de muestreo. Rev. Epidem. Med. Prev, (1), 3-7.

Cevallos, D. (2014). Uso del celular como medio de pago en Ecuador (Tesis de Grado). Universidad San Francisco de Quito, Ecuador.

Grijalva, E. (2014). Dinero Electrónico como Herramienta de Inclusión Financiera en el Ecuador (Tesis de Grado). Universidad San Francisco de Quito, Ecuador.

Jaime, E. (2014). El Dinero Electronico en el Ecuador. RES NON VERBA, 139-146.

Jeftanovic, P. (2001). El dinero electronico y la politica monetaria. Pharos, 8(2), 89-98.

Legeard, N. (2011). Nueva arquitectura financiera para el desarrollo en América Latina y Ecuador. Universitas, Revista de Ciencias Sociales y Humanas, (14), 45-73.

Méndez, S. (2015). Ecuador 2015: Análisis y propuesta de políticas públicas junto a alternativas de líquidez en contexto. FCSHOPINA, 86, 1-9.

Miranda, A. (2015). El Dinero Virtual y sus implicaciones para el Ecuador (Tesis de Grado). Universidad San Francisco de Quito, Ecuador. 
Quiroga, R. (2016). Dinero Electronico. (OMNIA, Entrevistador)

Santomá, J. (2001). Todo lo que debería saber sobre el Dinero Electrónico. Revista de Antiguos Alumnos, 82, 12 - 22.

UDLA. (2016). UDLA. Recuperado el 2017 de 01 de 29, de UDLA: http://www.udla.edu.ec/2016/10/17/dineroelectronico/

Valencia, F. (2015). Sistema de Dinero Electronico, un medio de pago al alcance de todos. Boletín, 60(4), 255-269. 